\title{
Implicación y motivación hacia los deberes escolares en los estudiantes de Primaria según el rendimiento académico y el curso
}

\author{
Antonio Valle, Bibiana Regueiro, Iris Estévez, Isabel Piñeiro, Susana \\ Rodríguez, y Carlos Freire \\ Universidad de A Coruña (España)
}

\begin{abstract}
Este trabajo pretende analizar si existen o no diferencias estadísticamente significativas en algunas variables motivacionales y de implicación en los deberes escolares, en función del curso y del nivel de rendimiento académico. La muestra se compone de 535 participantes de $4^{\circ}, 5^{\circ}$ y $6^{\circ}$ de Educación Primaria (de 9 a 13 años). Según el rendimiento académico, los resultados de la presente investigación indican que los alumnos con mayor rendimiento no sólo realizan más cantidad de deberes sino que además gestionan mejor el tiempo que dedican a ellos, los perciben más útiles e, incluso, están más motivados intrínsecamente hacia los mismos. Respecto del curso, los resultados demuestran que la actitud hacia los deberes escolares empeora y la motivación intrínseca e interés por los mismos disminuye a medida que los estudiantes avanzan en la escolaridad. También a medida que avanzan de curso los estudiantes dedican más tiempo a la realización de los deberes y la gestión de ese tiempo va siendo cada vez peor.
\end{abstract}

Palabras clave: Implicación en los deberes escolares, motivación hacia los deberes escolares, rendimiento académico, Educación Primaria.

Homework involvement and motivation in elementary school students according to academic achievement and grade. This paper analyzes statistically significant differences in some motivational and involvement in homework variables, depending on the course and the level of academic achievement. The sample consists of 535 participants of 4th, 5th and 6th Primary Education (from 9-13 years old). According to the academic performance, the results of this research indicate that students with higher academic achievement do more homework, manage better the time spent on them, perceive them more useful and even they are more motivated intrinsically toward them. Regarding the grade level, the results show that the attitude toward homework and intrinsic motivation and interest for homework decline as students advance to higher grade levels. Also as they advance, they spend more time doing homework and the time management becomes increasingly worse.

Keywords: Homework involvement, homework motivation, academic achievement, Elementary School.

Correspondencia: Antonio Valle. Departamento de Psicología Evolutiva y de la Educación. Universidad de A Coruña. Campus de Elviña, s/n. C.P.: 15071. A Coruña (España). E-mail: vallar@udc.es 
Los deberes escolares han sido siempre un tema de constante actualidad y han generado numerosos debates, en ocasiones más viscerales que fundamentados en investigaciones serias y rigurosas en torno a este tema. Si bien la investigación existente sobre los deberes ha estudiado un amplio número de relaciones entre diversas variables, la principal preocupación siempre ha estado centrada en si hacer deberes era o no beneficioso para el aprendizaje y el rendimiento académico de los estudiantes. Aunque se han producido avances importantes en la investigación, hay numerosas lagunas que a día de hoy todavía persisten. Es, por tanto, un tema enormemente controvertido y todavía no se tiene muy claro cómo, cuándo y cuántos deberes escolares deben ser prescritos a los estudiantes con el objetivo de que provoquen en ellos los mejores beneficios desde el punto de vista educativo.

El tiempo dedicado a los deberes ha sido una de las variables que más se ha tenido en cuenta a la hora de explicar los beneficios de los deberes escolares. Sin embargo, y a pesar de que muchos autores afirman que emplear tiempo en los deberes tiene efectos positivos en los logros académicos (Olympia, Sheridan, Jenson, y Andrews, 1994) y de que muchas de las investigaciones realizadas encuentran evidencia empírica de una relación significativa y positiva entre tiempo dedicado a los deberes y rendimiento, los resultados de algunas investigaciones actuales parecen discutir dicha relación. De hecho, muchos de los trabajos realizados sobre ello sólo encuentran una débil relación entre el tiempo dedicado a los deberes, el aprendizaje de los estudiantes y el rendimiento (Senechal y LeFevre, 2002). Por lo tanto, el tiempo invertido por el alumno en los deberes escolares no es en sí mismo una garantía de su compromiso en la realización de deberes asignados (Rosário, Mourão, Núñez, y Solano, 2008). El esfuerzo invertido en los deberes ha demostrado tener mayor influencia en el rendimiento académico que el tiempo dedicado a los deberes (Trautwein, Lüdtke, Kastens, y Köller, 2006). De esta forma, más que el tiempo como tal lo importante es el proceso de realización y el aprovechamiento real del tiempo dedicado, ya que cuanto mayor sea ese aprovechamiento, mayor será el rendimiento académico.

Si bien no existe consenso entre la relación cantidad de tiempo dedicado a la realización de los deberes y rendimiento, sí parece claro que cuantos más deberes escolares se realicen mayores serán sus beneficios en el logro académico. Sin embargo, existen diferencias significativas en cuanto a la cantidad de deberes realizados en aquellos alumnos con rendimiento alto y con rendimiento bajo: se observa un aumento progresivo en la cantidad de deberes realizados por los estudiantes a medida que sus niveles de rendimiento académico son más altos.

Ante el desafío de rebajar el nivel de fracaso escolar, es muy importante saber las situaciones en las cuales los deberes escolares benefician el rendimiento académico así como conocer aquellas otras situaciones en las que los deberes escolares perjudican dicho rendimiento. Parte de estas controversias tienen una posible explicación en la manera en 
la que los estudiantes maximizan los beneficios del tiempo que dedican a la realización de los deberes y la cantidad óptima de los mismos, según distintas variables como la edad, materia académica, conocimientos previos, habilidades de estudio y/o también la motivación y actitud hacia los deberes, entre otras. Los resultados de varias investigaciones indican que el tipo de motivación de los estudiantes ante una tarea se relaciona con la calidad de su implicación (Ryan y Deci, 2000 Aunque las razones que guían la conducta académica son múltiples y diversas (Valle et al., 2015), el tipo de motivos o de razones que tienen los estudiantes para hacer los deberes, junto con el grado de interés y la utilidad percibida que tienen de ellos inciden en su grado de implicación (cantidad de deberes realizados, tiempo dedicado a ellos y aprovechamiento de ese tiempo) y, consecuentemente, en su rendimiento académico (Pan et al., 2013;)

Un amplio número de trabajos analizaron las relaciones de las variables motivacionales con el rendimiento (Rodríguez et al., 2014; Rosário et al., 2009) pero, a pesar de ello, la investigación en función de la edad resulta escasa. Algunas investigaciones sugieren que la motivación disminuye a medida que se avanza de curso (Hong, Peng, y Rowell, 2009; Trautwein et al., 2006; Wigfield et al., 1997). Así, conforme los estudiantes cursan niveles más altos, tiene menos motivación intrínseca hacia a los deberes y los percibe menos útiles.

En base a estos planteamientos, el principal propósito de este trabajo es comprobar si hay diferencias en la implicación en los deberes escolar y la motivación hacia los mismos según el curso y el nivel de rendimiento académico de los estudiantes.

\section{MÉTODO}

\section{Participantes}

En el estudio participan 535 estudiantes pertenecientes a cuatro centros públicos de Educación Primaria de la provincia de A Coruña. De edades comprendidas entre los 9 y los 13 años $(M=10.32 ; D T=0.99)$, el $49.3 \%$ ( $N=264)$ son chicos y el $50.7 \%$ $(N=271)$ son chicas. En cuanto al curso, el 40.4\% ( $N=216)$ cursaban $4^{\circ}$ curso de Educación Primaria, el 35.1\% $(N=188)$ cursaban $5^{\circ}$ curso de Educación Primaria y el 24.5\% $(N=131)$ cursaban $6^{\circ}$ curso de Educación Primaria.

\section{Instrumentos}

Para poder realizar este estudio, se empleó como instrumento de medida la Encuesta sobre los Deberes Escolares (EDE) para medir las variables vinculadas con la implicación en los deberes escolares y la motivación. La Encuesta sobre los Deberes Escolares evalúa distintas dimensiones sobre la eficacia de los deberes para el aprendizaje y rendimiento académico de los alumnos (véase, p. e. Núñez, Suárez, Cerezo et al., 2015; 
Núñez et al., 2015; Pan et al., 2013; Rosário et al., 2009; Valle et al., 2015; Valle et al., 2015).

La estimación del número de deberes realizados por los alumnos se obtuvo mediante las respuestas a un ítem relativo a la cantidad de deberes realizados habitualmente, utilizando para ello una escala tipo likert con cinco alternativas ( 1 = ninguno, 2 = algunos, 3 = la mitad, 4 = casi todos, $5=$ todos $)$.

Para la medida del tiempo diario dedicado a la realización de los deberes, los estudiantes respondieron a tres ítems (en general, en una semana típica, en un fin de semana típico) con la formulación general “¿Cuánto tiempo sueles dedicar a la realización de los deberes?", siendo las opciones de respuesta $1=$ menos de 30 minutos, $2=$ de 30 minutos a una hora, $3=$ de una hora a hora y media, $4=$ de hora y media a dos horas, 5 = más de dos horas.

En cuanto al aprovechamiento del tiempo dedicado a los deberes, se midió a través de las respuestas a tres ítems (en general, en una semana típica, en un fin de semana típico) en los que se les pedía que indicaran el nivel de aprovechamiento del tiempo dedicado habitualmente a los deberes, utilizando para ello la siguiente escala: 1 = lo desaprovecho totalmente (me distraigo constantemente con cualquier cosa), $2=10$ desaprovecho más de lo que debiera, $3=$ regular, $4=$ lo aprovecho bastante, $5=10$ aprovecho totalmente (me concentro y hasta terminar no pienso en otra cosa).

La información sobre los motivos, intereses y percepciones que tienen los estudiantes de los deberes escolares abarcó las siguientes variables: a) motivación intrínseca hacia los deberes; b) interés por los deberes; c) actitud hacia deberes y d) percepción de utilidad de los deberes. Cada una de estas dimensiones está integrada por varios ítems con una escala tipo Likert ( $1=$ totalmente falso, hasta $5=$ totalmente cierto).

Finalmente, la evaluación del rendimiento académico se obtuvo mediante las calificaciones académicas finales obtenidas por los alumnos participantes en Lengua Española, Lengua Gallega, Lengua Inglesa, Conocimiento del Medio y Matemáticas. El rendimiento medio se calculó a través del promedio de las calificaciones en las cinco materias mencionadas. Los tres niveles de rendimiento académico (bajo, medio y alto) se establecieron en base a los siguientes criterios: rendimiento bajo (puntuaciones inferiores al percentil 25), rendimiento medio (puntuaciones entre los percentiles 25 y 75) y rendimiento alto (puntuaciones superiores al percentil 75).

\section{Procedimiento}

Los datos referidos a las variables objeto de estudio fueron recogidos durante el horario escolar por los autores del trabajo durante el segundo trimestre del curso académico 2014-15, previo consentimiento de la dirección de los centros y de los padres de los alumnos, indicándoles en todo momento que la única finalidad era el llevar a cabo 
una investigación sobre los deberes escolares y que, por lo tanto, los datos tenían un carácter totalmente confidencia.

\section{Análisis de datos}

Con el fin de dar respuesta a los objetivos del estudio, en un primero momento se realizaron análisis multivariados de la varianza (MANOVA) tomando como factores el rendimiento académico (con tres niveles) y el curso (con tres niveles), y como variables dependientes el interés por los deberes, la motivación intrínseca hacia los deberes, la percepción de utilidad de los deberes, la actitud hacia los deberes, , la cantidad de deberes realizados, el tiempo dedicado a los deberes y el aprovechamiento del tiempo dedicado a los deberes. Como medida del tamaño del efecto se ha utilizado el coeficiente eta-cuadrado parcial $\left(\eta_{\mathrm{p}}{ }^{2}\right)$, ya que es uno de los procedimientos más utilizados habitualmente dentro de la investigación educativa (Sun, Pan, y Wang, 2010). Para la interpretación de los tamaños del efecto se utiliza el criterio establecido en el trabajo clásico de Cohen (1988), en base al cual, un efecto es pequeño cuando $\eta_{\mathrm{p}}{ }^{2}=.01(d=.20)$, el efecto es medio cuando $\eta_{\mathrm{p}}{ }^{2}=.059$ $(d=.50)$ y el tamaño del efecto es grande si $\eta_{\mathrm{p}}{ }^{2}=.138(d=.80)$.

\section{RESULTADOS}

Los resultados del MANOVA indican que sí hay diferencias estadísticamente significativas en el conjunto de variables vinculadas con la motivación y con la implicación en los deberes escolares en función de los distintos niveles de rendimiento académico (Lambda de Wilks $\left.=.821, F_{(14,1040)}=7.71, p<.001, \eta_{\mathrm{p}}{ }^{2}=.094\right)$, con un tamaño del efecto cercano a grande.

En lo que respecta a los resultados referidos a cada variable dependiente tomada de forma individual, existen diferencias estadísticamente significativas, en función del nivel de rendimiento académico, en la motivación intrínseca hacia los deberes escolares $\left(F_{(2,526)}=7.07, p<.001, \eta_{\mathrm{p}}{ }^{2}=.026\right)$, en la percepción de utilidad de los deberes $\left(F_{(2,526)}=3.13\right.$, $\left.p<.05, \eta_{\mathrm{p}}{ }^{2}=.012\right)$, en la cantidad de deberes realizados $\left(F_{(2,526)}=34.37, p<.001, \eta_{\mathrm{p}}{ }^{2}=.116\right)$ $\mathrm{y}$ en el aprovechamiento del tiempo dedicado a los deberes $\left(F_{(2,526)}=11.28, p<.001\right.$, $\left.\eta_{\mathrm{p}}{ }^{2}=.041\right)$. Sólo en el caso de la cantidad de deberes realizados, el tamaño del efecto se aproxima a grande. En cambio, no hay diferencias estadísticamente significativas, en función del nivel de rendimiento académico, en el interés por los deberes $\left(F_{(2,526)}=0.84\right.$, $\left.p=.433, \eta_{\mathrm{p}}{ }^{2}=.003\right)$, en la actitud hacia los deberes $\left(F_{(2,526)}=2.69, p=.069, \eta_{\mathrm{p}}{ }^{2}=.010\right)$ y en el tiempo dedicado a los deberes $\left(F_{(2,526)}=0.20, p=.820, \eta_{\mathrm{p}}{ }^{2}=.001\right)$. Los tamaños del efecto son pequeños.

A medida que el rendimiento académico es más alto (ver tabla 1), también aumenta progresivamente la motivación intrínseca hacia los deberes, la percepción de utilidad de esos deberes, la cantidad de deberes realizados y el aprovechamiento del 
VALLE et al. Implicación y motivación hacia los deberes escolares en los estudiantes de Primaria...

tiempo dedicado a los deberes. Por el contrario, el interés, la actitud y el tiempo dedicado a los deberes no varían significativamente en función de los distintos niveles de rendimiento académico.

Tabla 1. Estadísticos descriptivos (media, desviación típica) correspondientes a las variables vinculadas con la motivación y con la implicación en los deberes escolares en función del curso y de los niveles de rendimiento académico

\begin{tabular}{|c|c|c|c|c|c|c|c|c|}
\hline & \multicolumn{2}{|c|}{$4^{\circ} \mathrm{EP}$} & \multicolumn{2}{|c|}{$5^{\circ} \mathrm{EP}$} & \multicolumn{2}{|c|}{$6^{\circ} \mathrm{EP}$} & \multicolumn{2}{|c|}{ Total } \\
\hline & $M$ & $D T$ & $M$ & $D T$ & $M$ & $D T$ & $M$ & $D T$ \\
\hline \multicolumn{9}{|c|}{ Motivación intrínseca deberes escolares } \\
\hline Rend. bajo & 4.29 & 0.76 & 4.11 & 0.78 & 3.88 & 0.70 & 4.10 & 0.76 \\
\hline Rend. medio & 4.48 & 0.56 & 4.31 & 0.66 & 4.07 & 0.57 & 4.32 & 0.61 \\
\hline Rend. alto & 4.60 & 0.39 & 4.22 & 0.53 & 4.24 & 0.56 & 4.40 & 0.51 \\
\hline Total & 4.49 & 0.56 & 4.22 & 0.67 & 4.06 & 0.62 & 4.29 & 0.64 \\
\hline \multicolumn{9}{|c|}{ Interés deberes escolares } \\
\hline Rend. bajo & 4.08 & 0.95 & 3.64 & 1.07 & 3.22 & 0.99 & 3.65 & 1.06 \\
\hline Rend. medio & 3.98 & 0.91 & 3.76 & 1.02 & 3.45 & 0.90 & 3.78 & 0.96 \\
\hline Rend. alto & 4.20 & 0.81 & 3.58 & 0.86 & 3.60 & 0.99 & 3.86 & 0.91 \\
\hline Total & 4.07 & 0.89 & 3.67 & 0.99 & 3.42 & 0.96 & 3.77 & 0.97 \\
\hline \multicolumn{9}{|c|}{ Actitud deberes escolares } \\
\hline Rend. bajo & 3.94 & 0.98 & 3.28 & 1.10 & 2.69 & 1.03 & 3.30 & 1.14 \\
\hline Rend. medio & 3.50 & 1.00 & 3.15 & 1.05 & 2.74 & 0.80 & 3.20 & 1.01 \\
\hline Rend. alto & 3.58 & 0.87 & 2.80 & 0.81 & 2.76 & 0.83 & 3.15 & 0.93 \\
\hline Total & 3.61 & 0.96 & 3.09 & 1.01 & 2.73 & 0.88 & 3.21 & 1.02 \\
\hline \multicolumn{9}{|c|}{ Percepc. utilidad deberes escolares } \\
\hline Rend. bajo & 4.49 & 0.92 & 4.24 & 1.26 & 3.87 & 1.09 & 4.21 & 1.14 \\
\hline Rend. medio & 4.57 & 0.86 & 4.45 & 0.70 & 4.04 & 0.90 & 4.41 & 0.85 \\
\hline Rend. alto & 4.70 & 0.57 & 4.29 & 0.78 & 4.39 & 0.77 & 4.50 & 0.71 \\
\hline Total & 4.60 & 0.78 & 4.34 & 0.93 & 4.08 & 0.94 & 4.38 & 0.90 \\
\hline \multicolumn{9}{|c|}{ Cantidad de deberes realizados } \\
\hline Rend. bajo & 4.58 & 0.77 & 4.17 & 1.09 & 3.92 & 0.97 & 4.22 & 1.00 \\
\hline Rend. medio & 4.66 & 0.73 & 4.79 & 0.44 & 4.71 & 0.46 & 4.71 & 0.59 \\
\hline Rend. alto & 4.80 & 0.57 & 4.91 & 0.44 & 4.89 & 0.32 & 4.86 & 0.48 \\
\hline Total & 4.69 & 0.69 & 4.63 & 0.77 & 4.52 & 0.75 & 4.63 & 0.73 \\
\hline \multicolumn{9}{|c|}{ Tiempo dedicado a los deberes } \\
\hline Rend. bajo & 2.07 & 1.10 & 2.60 & 1.18 & 2.90 & 1.19 & 2.53 & 1.20 \\
\hline Rend. medio & 2.26 & 0.99 & 2.59 & 1.15 & 2.71 & 1.01 & 2.48 & 1.07 \\
\hline Rend. alto & 2.13 & 1.15 & 3.00 & 1.19 & 2.64 & 1.20 & 2.53 & 1.23 \\
\hline Total & 2.18 & 1.07 & 2.71 & 1.18 & 2.75 & 1.12 & 2.51 & 1.15 \\
\hline \multicolumn{9}{|c|}{ Aprovechamiento del tiempo dedicado a los deberes } \\
\hline Rend. bajo & 4.22 & 0.99 & 3.69 & 1.14 & 3.35 & 1.05 & 3.75 & 1.12 \\
\hline Rend. medio & 4.18 & 0.82 & 4.21 & 0.89 & 3.93 & 0.83 & 4.13 & 0.85 \\
\hline Rend. alto & 4.38 & 0.88 & 4.05 & 0.70 & 4.28 & 0.66 & 4.25 & 0.79 \\
\hline Total & 4.26 & 0.88 & 4.00 & 0.95 & 3.85 & 0.93 & 4.07 & 0.93 \\
\hline
\end{tabular}

En lo que respecta a las diferencias en las variables relacionadas con la motivación e implicación en los deberes escolares en función del curso, los resultados del MANOVA indican que hay diferencias estadísticamente significativas en el conjunto de estas variables $\left(\lambda_{\text {Wilks }}=.799, F_{(14,1040)}=8.81, p<.001, \eta_{\mathrm{p}}{ }^{2}=.106\right)$. El tamaño del efecto es muy próximo a grande. Si nos centramos en cada variable dependiente en particular, los resultados indican que hay diferencias estadísticamente significativas en función del curso en la motivación intrínseca hacia los deberes escolares $\left(F_{(2,526)}=17.04, p<.001, \eta_{\mathrm{p}}{ }^{2}=.061\right)$, en el interés por los deberes $\left(F_{(2,526)}=20.29, p<.001, \eta_{\mathrm{p}}{ }^{2}=.072\right)$, en la actitud hacia los 
deberes $\left(F_{(2,526)}=39.99, p<.001, \eta_{\mathrm{p}}{ }^{2}=.132\right)$, en la percepción de utilidad de los deberes $\left(F_{(2,526)}=12.07, p<.001, \eta_{\mathrm{p}}{ }^{2}=.044\right)$, en el tiempo dedicado a los deberes $\left(F_{(2,526)}=15.89\right.$, $\left.p<.001, \eta_{\mathrm{p}}^{2}=.057\right)$ y en el aprovechamiento del tiempo dedicado a los deberes $\left(F_{(2,526)}=8.86, p<.001, \eta_{\mathrm{p}}{ }^{2}=.033\right)$. Únicamente, esas diferencias no son significativas en la cantidad de deberes realizados $\left(F_{(2,526)}=2.47, p=.085, \eta_{\mathrm{p}}{ }^{2}=.009\right)$. En el caso de la actitud hacia los deberes, el tamaño del efecto es grande, mientras que en el caso del interés por los deberes, la motivación intrínseca hacia los deberes y el tiempo dedicado a los deberes, el tamaño del efecto es medio.

Tal y como puede observarse en la tabla 1, según van avanzando los estudiantes de curso, disminuye progresivamente su motivación intrínseca hacia los deberes, su interés y su actitud hacia los deberes, así como la percepción de utilidad de estos deberes. Al mismo tiempo, también se incrementa el tiempo que dedican a los deberes, pero el aprovechamiento de ese tiempo disminuye. Mientras tanto, no hay diferencias significativas en función del curso en la cantidad de deberes realizados.

\section{DISCUSIÓN}

Con el presente estudio se pretendió aportar información sobre la investigación de los deberes escolares. Por un lado, los resultados de este trabajo ponen de manifiesto que los niveles más altos de rendimiento académico están asociados con una mayor cantidad de deberes realizados, con un mejor aprovechamiento del tiempo dedicado a los deberes, con una mayor motivación intrínseca hacia los deberes escolares y con una mayor percepción de utilidad de los deberes. Ni el interés, ni la actitud, ni el tiempo dedicado a los deberes varían significativamente en función del rendimiento académico.

Del mismo modo y si tenemos en cuenta que la motivación está considerada como una parte esencial en el proceso de los deberes escolares y, a su vez, los deberes escolares juegan un papel crítico en el desarrollo de la motivación de logro del alumnado (Bempechat et al., 2011) resulta preocupante el hallazgo del presente estudio, el cual indica que a medida que los estudiantes avanzan de curso disminuye su motivación intrínseca, su interés y su actitud hacia los deberes. Estos resultados se encuentran, por tanto, en consonancia con aquellos estudios previos que indican que la motivación disminuye a medida que se avanza de curso (Hong et al., 2009; Trautwein et al., 2006; Wigfield et al., 1997). De esta forma todo parece indicar que conforme el estudiante cursa niveles más altos tiene menos motivación intrínseca hacia a los deberes y los percibe menos útiles (Regueiro, Suárez, Valle, Núñez, y Rosário, 2015).

Asimismo, otro resultado destacado es que según van avanzando los estudiantes de $4^{\circ}$ a $6^{\circ}$ curso de Primaria se produce un aumento progresivo del tiempo que dedican a hacer los deberes escolares y, sin embargo, el aprovechamiento que hacen de ese tiempo va siendo cada vez menor. Esta disminución del aprovechamiento del tiempo dedicado a 
los deberes coincide con los resultados de otros trabajos realizados con estudiantes de Primaria (Valle et al., 2015). Además, debido a que la exigencia académica es mayor, la importancia de completar los deberes se incrementa a medida que los estudiantes avanzan en la escuela, lo que los convierte en algo imprescindible para lograr el éxito académico (Zimmerman y Kitsantas, 2005).

Es importante destacar que estos aumentos y disminuciones según se avanza de curso siguen una tendencia algo diferente dependiendo del nivel de rendimiento académico al que se haga referencia. En el caso del nivel de rendimiento alto, los mayores incrementos o disminuciones en la mayor parte de las variables suelen producirse en $5^{\circ}$ curso. En $6^{\circ}$ curso, el buen rendimiento está asociado a un incremento también de las condiciones motivacionales y actitudinales relacionadas con los deberes escolares.

De esta forma, y en consonancia con gran parte de la investigación previa, los datos aportados por este estudio aportan evidencia de la asociación positiva entre la cantidad de deberes realizados y el rendimiento académico obtenido. Este resultado parece muy claro en el caso de estudiantes de Secundaria y Bachillerato (Cooper, Robinson, y Patall, 2006), aunque también se ha encontrado en estudiantes de Primaria (Pan et al., 2013). En una revisión llevada a cabo por Cooper (1989) de 120 estudios empíricos sobre los efectos de los deberes escolares se encontraron 50 estudios que muestran correlaciones entre cantidad de tiempo que los estudiantes dedican a los deberes y sus logros escolares. En 43 de ellos, se observa que los estudiantes que hacen más deberes tienen mejores resultados académicos, mientras que sólo 7 de los estudios muestran relaciones negativas entre ambas variables. Otro aspecto destacado es que para los estudiantes de Primaria, la relación entre cantidad de deberes realizados y logros académico es casi nula. En cambio, esa relación va siendo cada vez más fuerte y positiva según se va avanzando hacia Secundaria. Por tanto, parece demostrado que los estudiantes que completan sus deberes escolares tienen unas mejores calificaciones académicas que aquellos que no los completan (Cooper, 1989; Núñez et al., 2015; Trautwein, Köller, Schmitz, y Baumert, 2002; Valle et al., 2015). Este resultado apoya, sin duda, la opinión de quienes defienden los deberes escolares como un instrumento útil y fundamental en la realidad escolar (Trautwein et al., 2002).

En síntesis, a pesar de que los deberes cobran importancia por el aumento de la exigencia académica, los datos aportados por el presente estudio y en consonancia con estudios previos sugieren que las variables tanto motivacionales como las de implicación en los deberes escolares decrecen a medida que el alumnado va avanzando de curso.

Evidentemente algunas cuestiones arrojadas de las conclusiones de este trabajo deberían de ser analizadas, tanto desde el campo de la investigación como también desde la práctica educativa en el aula. Entre ellas destaca el descenso del interés, motivación y actitud de los estudiantes hacia los deberes escolares a medida que suben de curso. Precisamente, una posible explicación puede ser que a medida que ascienden de curso los 
estudiantes perciban como menos importante la realización de los deberes prescritos por el profesor, o quizás, este descenso también podría deberse a que en sus vidas comienzan a emerger inquietudes y tareas vitales que ocupan cada vez más su tiempo y su pensamiento. Y por lo que parece, dentro de estas prioridades e inquietudes va teniendo cada vez menos protagonismo lo que se enseña y aprende en las aulas $y$, consecuentemente, los deberes escolares y la motivación e interés por realizarlos.

Dentro de las limitaciones de este estudio, es necesario indicar que la evaluación de algunas variables mediante autoinformes así como el reducido número de ítems para medir dichas variables constituye una de las limitaciones que hay que tener presente a la hora de analizar las aportaciones derivadas del mismo. También es necesario señalar que se trata de un estudio transversal, con lo cual no es posible establecer relacionales causales entre las variables (Inglés et al., 2014). Por eso, un estudio longitudinal podría servir para clarificar las posibles relaciones causales entre algunas variables (Estévez, Inglés, y Martínez-Monteagudo, 2013). Por otro lado, a pesar de que este trabajo sólo se centró en el nivel del alumno, en el futuro será necesario considerar otros niveles de análisis (nivel de clase, nivel de centro educativo, etc.), ya que los resultados, tal y como plantean Trautwein et al. (2002), pueden ser muy diferentes.

\section{Agradecimientos}

Este trabajo se ha desarrollado gracias a la financiación del proyecto de investigación EDU2013-44062-P, perteneciente al Plan Estatal de Investigación Científica y Técnica y de Innovación 2013-2016 (MINECO) y al financiamiento recibido por una de las autoras en el Programa FPU del Ministerio de Educación, Cultura y Deporte.

\section{REFERENCIAS}

Bempechat, J., Li, J., Neier, S.M., Gillis, C.A., y Holloway, S.D. (2011). The homework experience: Perceptions of low-income youth. Journal of Advanced Academics, 22(2), 250-278.

Cohen, J. (1988). Statistical power analysis for the behavioral sciences ( $2^{\mathrm{a}}$ ed.). Hillsdale, NJ: Erlbaum.

Cooper, H. (1989). Synthesis of research on homework. Educational Leadership, 47(3), 85-91.

Cooper, H., Robinson J.C., y Patall, E.A. (2006). Does homework improve academic achievement? A synthesis of research, 1987-2003. Review of Educational Research, 76(1), 1-62.

Estévez, E., Inglés, C.J., y Martínez-Monteagudo, M.C. (2013). School aggression: Effects of classroom environment, attitude to authority and social reputation among peers. European Journal of Investigation in Health, Psychology and Education, 3(1), 15-28.

Hong, E., Peng, Y., y Rowell, L.L. (2009). Homework self-regulation: Grade, gender, and achievement-level differences. Learning and Individual Differences, 19(2), 269-276.

Inglés, C.J., Torregrosa, M.S., García-Fernández, J.M., Martínez-Monteagudo, M.C., Estévez, E., y Delgado, B. (2014). Conducta agresiva e inteligencia emocional en la adolescencia. European Journal of Education and Psychology, 7(1), 29-41.

Núñez, J.C., Suárez, N., Cerezo, R., González-Pienda, J.A., Rosário, P., Mourao, R., y Valle, A. (2015). Homework and academic achievement across Spanish Compulsory Education. Educational Psychology, 35(6), 726-746. 
Núñez, J.C., Suárez, N., Rosário, P., Vallejo, G., Cerezo, R., y Valle, A. (2015). Teachers’ feedback on homework, homework-related behaviors and academic achievement. The Journal of Educational Research, 108(3), 204-216.

Olympia, D.E, Sheridan, S.M, Jenson, W.R, y Andrews D. (1994). Using student-managed interventions to increase homework completion and accuracy. Journal of Applied Behavior Analysis, 27, 85-99.

Pan, I., Regueiro, B., Ponte, B., Rodríguez, S., Piñeiro. I., y Valle, A. (2013). Motivación, implicación en los deberes escolares y rendimiento académico. Aula Abierta, 41(3), 1322.

Regueiro, B., Suárez, N., Valle, A., Núñez, J.C., y Rosário, P. (2015). La motivación e implicación en los deberes escolares a lo largo de la escolaridad obligatoria. Revista de Psicodidáctica, 20(1), 47-63.

Rodríguez, S., Regueiro, B., Blas, R., Valle, A., Piñeiro., I., y Cerezo, R. (2014). Teacher selfefficacy and its relationship with students' affective and motivational variables in higher education. European Journal of Education and Psychology, 7(2), 107-120.

Rosário, P., Mourão, R., Baldaque, M., Nunes, T., Núñez, J.C., González-Pienda, J.A., Cerezo, R., y Valle, A. (2009). Tareas para casa, autorregulación del aprendizaje y rendimiento en matemáticas. Revista de Psicodidáctica, 14, 179-192.

Rosário, P., Mourão, R., Núñez, J.C., y Solano, P. (2008). Homework and Self-Regulated Learning (SRL) at issue: findings and future trends. En A. Valle, J.C. Núñez, R.G. Cabanach, J.A. González-Pienda, y S. Rodríguez (Eds.), Handbook of instructional resources and their applications in the classroom (pp. 123-134). Nueva York: Nova Science Publishers.

Ryan, E.M., y Deci, E.L. (2000). Self-determination theory and the facilitation of motivation, social development, and well-being. American Psychologist, 55, 68-78.

Senechal, M., y LeFevre, J. (2002). Parental involvement in the development of children's reading skill: A five-year longitudinal study. Child Development, 73(2), 445-460.

Sun, S., Pan, W., y Wang, L.L. (2010). A comprehensive review of effect size reporting and interpreting practices in academic journals in education and psychology. Journal of Educational Psychology, 102, 989-1004.

Trautwein, U., Köller, O., Schmitz, B., y Baumert, J. (2002). Do homework assignments enhance achievement? A multilevel analysis of 7th grade mathematics. Contemporary Educational Psychology, 27, 26-50.

Trautwein, U., Lüdtke, O. Kastens, C., y Köller, O. (2006). Effort on homework in grades 5 through 9: Development, motivational antecedents, and the association with effort on class-work. Child Development, 77, 1094-1111.

Valle, A., Pan, I., Núñez, J.C., Rosário, P., Rodríguez, S., y Regueiro, B. (2015). Deberes escolares y rendimiento académico en Educación Primaria. Anales de Psicología, 31(2), 562-569.

Valle, A., Pan, I., Regueiro, B., Suárez, N., Tuero, E., y Rosário, P. (2015). Predicting approach to homework in primary school students. Psicothema, 27(4), 334-340.

Valle, A., Regueiro, B., Rodríguez, S., Piñeiro, I., Freire, C., Ferradás, M., y Suárez, N. (2015). Perfiles motivacionales como combinación de expectativas de autoeficacia y metas académicas en estudiantes universitarios. European Journal of Education and Psychology, $8(1), 1-8$.

Wigfield, A., Eccles, J.S., Yoon, K.S., Harold, R.D., Arbreton, A.J.A., Freedman-Doan, C., y Blumenfeld, P.C. (1997). Change in children's competence beliefs and subjective task values across the elementary school years: A 3-year study. Journal of Educational Psychology, 89(3), 451-469. 
VALLE et al. Implicación y motivación hacia los deberes escolares en los estudiantes de Primaria...

Zimmerman, B.J., y Kitsantas, A. (2005). Students' perceived responsibility and completion of homework: The role of self-regulatory beliefs and processes. Contemporary Educational Psychology, 30, 397-417.

Recibido: 16 de octubre de 2015

Recepción Modificaciones: 20 de noviembre de 2015

Aceptado: 30 de noviembre de 2015 